\title{
Descriptor for Hydrogen Evolution Catalysts Based on the Bulk Band Structure Effect
}

\author{
Qiunan Xu, Guowei Li, Yang Zhang, Qun Yang, Yan Sun,* and Claudia Felser*
}

Cite This: ACS Catal. 2020, 10, 5042-5048

Read Online

ABSTRACT: The vital role of electrocatalysts in determining the efficiency of renewable energy conversion inspired the uncovering of the relation between the catalytic efficiency and electronic structure, in which the volcano-type plot based on adsorption energies and d-band model has achieved great success. At the same time, catalysts with nontrivial topological electronic structures have received considerable attention because of their robust topological surface states and high-mobility electrons, which favor the electrons transfer processes in the heterogeneous catalysis reactions. Under the guidance of this theory, excellent catalysts were reported among

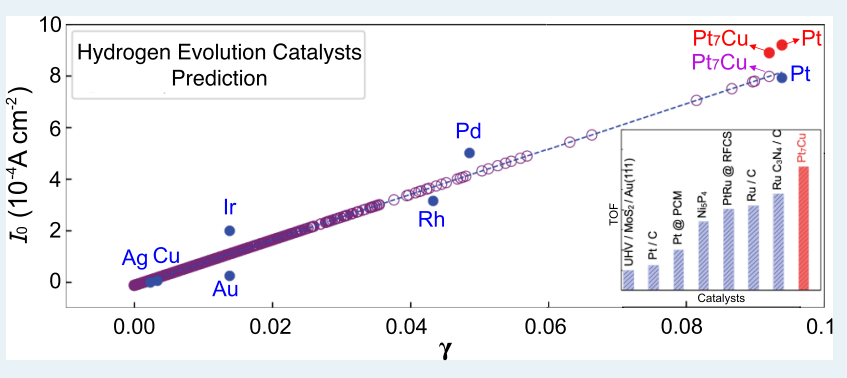
topological materials. Inspired by the current development of catalyst and topological materials, we tried to extract a pure intrinsic physical parameter, projected Berry phase (PBP), that only depends on the bulk electronic structure. Applying this parameter to the well-known nonmagnetic transition-metal electrocatalysts, we found a linear relationship between PBP and catalytic efficiency of hydrogen evolution reaction (HER) after considering the symmetry constraint. This can be used as a descriptor for the prediction and designing of promising catalysts for $\mathrm{HER}$, which is realized experimentally in $\mathrm{Pt}_{7} \mathrm{Cu}$ nanostructures. This work illustrates the importance of the pure bulk band structure effect on electrochemical activities and implies an effective way to understand the mechanism of HER catalysts.

KEYWORDS: hydrogen evolution catalysts, topological catalytic materials, projected Berry phase, bulk electronic structure, HER catalyst prediction

\section{INTRODUCTION}

Hydrogen has been considered as one of the most promising future carriers of renewable energy for powering electric vehicles and devices. Owing to the abundant water resources and the absence of any carbon-related side product, electrochemical water splitting is believed to be an ideal and sustainable process for hydrogen generation. Scalable and economical production requires electrocatalysts that cost less but retain as high activity as Pt. ${ }^{1}$ Thus, in the last few decades, the identification of effective physical parameters that are relevant to catalytic efficiency had become important for the understanding of the micro hydrogen evolution reaction (HER) mechanism and the search for high-efficiency catalysts. $^{2-14}$

Nowadays, the HER mechanism can be understood by three steps. Initially, the Volmer reaction transfer one electron to a proton to form adsorbed $\mathrm{H}$ on the catalyst surface $\left(\mathrm{H}^{+}+\mathrm{e}^{-}+\right.$ $* \rightarrow \mathrm{H}^{*}$, where $*$ denotes the active centers and $\mathrm{H}^{*}$ is the intermediate). The desorbing of $\mathrm{H}_{2}$ can be achieved by either Tafel reaction $\left(2 \mathrm{H}^{*} \rightarrow \mathrm{H}_{2}+2^{*}\right)$ or Heyrovsky reaction $\left(\mathrm{H}^{+}+\right.$ $\left.\mathrm{e}^{-}+\mathrm{H}^{*} \rightarrow \mathrm{H}_{2}+*\right)$. Apparently, this is a two-electron transfer process with $\mathrm{H}^{*}$ as the only one reaction intermediate. Thus, the overall HER reaction rate is closely related to the bond strength between the intermediate and the active sites, which is the theoretical basement of the d-band theory. ${ }^{8,9,15,16}$ Finally, a volcano-shaped form ${ }^{4,7,17,18}$ is obtained by plotting the exchanged current densities with the hydrogen adsorption free energy $\left(\Delta G_{\mathrm{H}}\right)$ at the equilibrium potential. It states that the metal-hydrogen bond should not be too strong or too weak. If the binding is too strong, the desorption step (Heyrovsky or Tafel) becomes difficult and limits the overall reaction rate; if the binding is too weak, the adsorption (Volmer) step will be the predominant mechanism and ratelimiting step. ${ }^{2,4,7,17,19,20}$ This has been commonly used as a "descriptor" for describing the hydrogen evolution activity and guiding the search of new catalysts. It should be noted that such a volcano-picture is based on the viewpoint of thermodynamics and overlook the kinetically determined barrier part. $^{21,22}$ This brings a lot of puzzles when merely using the descriptor $\Delta G_{\mathrm{H}} \approx 0$ to evaluate the efficiency of a catalyst. $^{23}$ Therefore, the kinetics part of the reaction which is

Received: December 23, 2019

Revised: $\quad$ March 31, 2020

Published: April 3, 2020 
governed by the transition state with the highest free energy should be taken into consideration when an overpotential is applied. ${ }^{24-26}$ However, the hydrogen binding energy near the equilibrium still provides an important indication for the searching of HER catalysts with high performance.

A further investigation of the traditional volcano plot found that both the adsorption energy and the metal-hydrogen bond strength can be related to simple physical descriptors such as work function ${ }^{4,10}$ and d-band center ${ }^{8,9}$ or d-band shape ${ }^{16}$ of catalysts. Because both the work function and the d-band center are physical parameters that are strongly dependent on the electronic structure, the efficiency of the catalyst should be understood from fundamental electrical properties. Though the chemical reaction of HER happens on the surface, the surface itself is strongly dependent on the bulk band structure. For example, the electrochemical performances of some catalysts are naturally much better than others, regardless of their morphology, defects, or even mass loading. Pt is such a catalyst with high intrinsic activity. ${ }^{4}$ Moreover, strategies such as introducing defects, doping, nanostructuring, and coupling with graphene ${ }^{19,27-29}$ could optimize the catalytic centers efficiently and boost the catalytic activities by orders of magnitude. In the last decade, with the observation of some high efficiency catalysts in topological materials, ${ }^{30-34}$ lots of efforts had been devoted to understanding the topological effect. It is believed that topological materials should be good candidates as high efficiency catalysts because of their robust surface states and high mobilities caused by linear band crossing. Very recently, the best catalyst $\mathrm{Pt}$ was proposed to be a $Z_{2}$ topological semimetal, ${ }^{35}$ which implies the possible relation between topological band structure and catalysis. However, it was also found that the catalytic efficiencies of the other similar $Z_{2}$ topological semimetals, ${ }^{35}$ such as Pt's neighbors $\mathrm{Au}$ and $\mathrm{Ag}$, are inferior to $\mathrm{Pt}$. Therefore, the quantum topological invariant would not be a good descriptor that connects the catalytic efficiency and topological band structure, and some noninteger quantities that can describe more details are needed when considering the intrinsic bulk band structure. This inspired us to think about the possibility of extracting an intrinsic physical parameter from the bulk electronic structure that is strongly related to the catalyst performance. In this work, we defined a parameter that only depend on the intrinsic electronic structure of an ideal bulk single crystal, and this parameter has a good linear relationship with the exchange current densities. This work may provide an effective way to understand the catalysis from fundamental intrinsic physical parameters of bulk electronic structure.

Our motivation is schematically shown in Figure 1. The HER occurs at the surfaces of catalysts, which are generally loaded on the cathode of the electrochemical system. Electrons are first injected into the catalyst, which then populates the valence band up to the highest occupied molecular orbital (HOMO, Fermi level). With a negative potential applied on the cathode, the electron energy can be governed and raise the Fermi level above the lowest unoccupied molecular orbital (LUMO) of the adsorbates $\left(\mathrm{H}^{+}\right)$. Thus, electrons can flow into the empty orbitals on $\mathrm{H}$ adsorbates and the reduction process (Volmer step) takes place. With this in mind, we can conclude that besides chemical stability and economical consideration, a good HER catalyst with high catalytic performance is controlled jointly by various physical characteristics, including electrical conductivity, carrier velocity, carrier mobility, adsorption energy, and so forth. ${ }^{36,37}$ All these physical

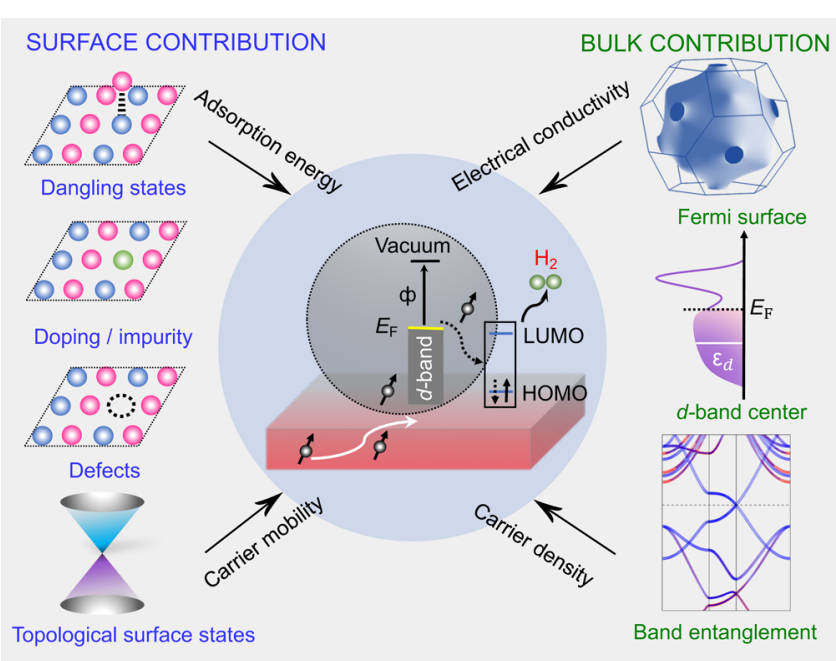

Figure 1. Motivation of this work. There is an assumption that the HER catalytic process is decided by the combined effect of electronic conductivity, carrier density, carrier mobility, adsorption energy, and so forth. The effects of the electronic structure can be classified into two groups: bulk and surface contributions. The bulk contribution mainly depends on the band entanglements and Fermi surface.

parameters are decided by electronic structures. Because the surface is smoothly connected with bulk, the surface states (both topological surface states and dangling bonds) are originally decided by the bulk electronic structure in some sense. The effect of the bulk structure to the other physical properties are normally separated into two contributions, Fermi surface and band entanglement. The Fermi surface contribution depends on lots of details, such as impurities, defects, scatterings, and so forth. In comparison, the band entanglement is a pure intrinsic contribution that only depends on the ideal band structure of a pure single crystal. Thus, it should be robust as long as the crystal structure is clean enough. Our goal in this work is to obtain a pure intrinsic bulk parameter that might be related to the catalytic process through exchange current density.

\section{RESULTS AND DISCUSSION}

Among the intrinsic parameters in solid-state crystals, the Berry phase and the Berry curvature of the electronic wave function have their special positions. They have a profound effect on topological and electrical properties in materials that are related to the carrier density, velocity, mobility, and so forth. ${ }^{38}$ The natural advantage of the Berry phase should make it an acceptable candidate to analyze the intrinsic effect of the electronic structure in the catalyst. However, because the Berry curvature is odd with respect to time reversal symmetry and even with respect to space inversion symmetry, there is no Berry phase (integral of Berry curvature across the whole reciprocal space) in the nonmagnetic system. Thus, the Berry curvature is forced to be zero at any point in a system with time reversal and space inversion symmetry. ${ }^{38}$ Because most of the HER catalysts are nonmagnetic compounds (such as the transition metals) with the highly symmetrical crystal structure, the strict symmetry requirement of the Berry phase strongly limits their application in catalysts.

From the definition of the Berry curvature 

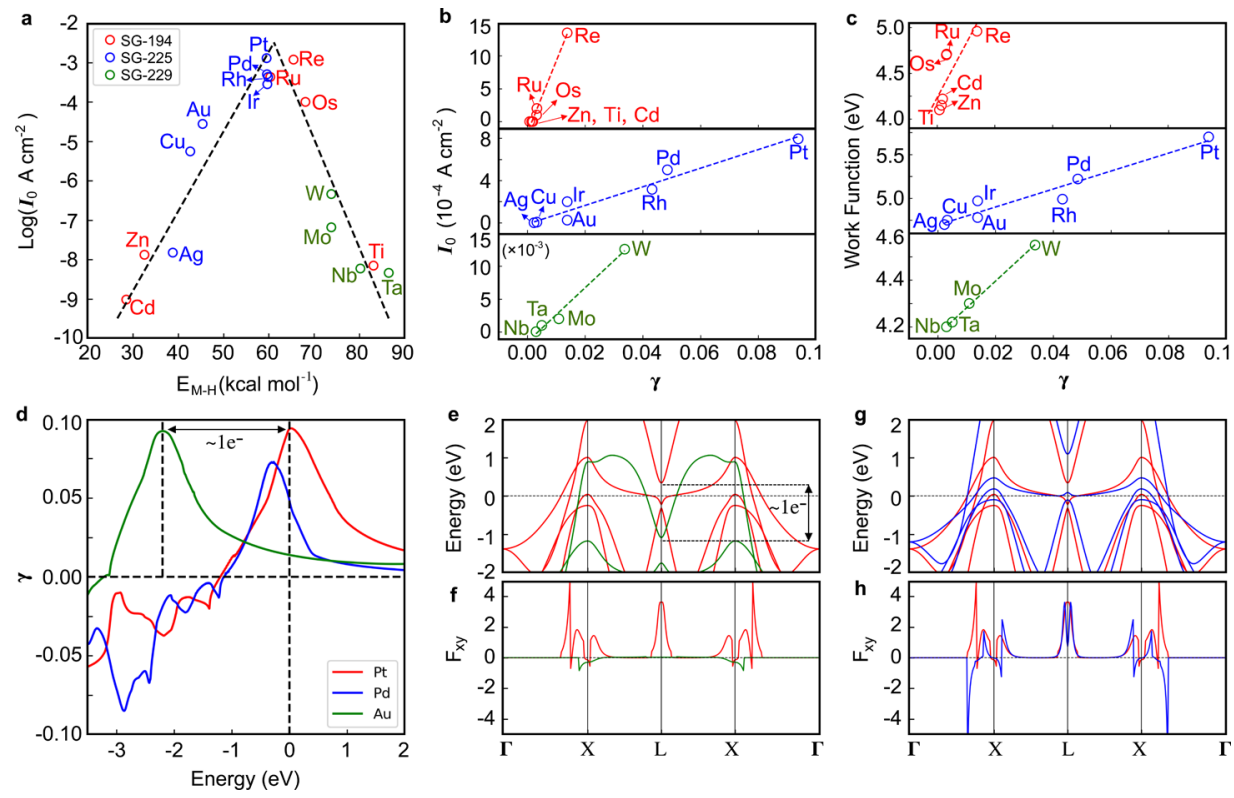

Figure 2. (a) Well-known volcano-type plot of exchange current density (logarithm form) for HER as a function of metal-hydrogen bond strength for the collected experimental data from the literature. ${ }^{4,39-41}$ Plot of (b) PBP vs the exchange current density (logarithm form) and (c) PBP vs the work function ${ }^{4,42}$ for HER have a good linear relation for the selected data after considering symmetry constraint. All the collected elements can be classified into three space groups. The red, blue and green circles in (a-c) represent space groups $P 6_{3} / m m c$ (no. 194), Fm $\overline{3} m$ (no. 225 ), and $I m \overline{3} m$ (no. 229), respectively. (d) Fermi-energy-dependent PBP for the three transition metals Pt, Au, and Pd. (e,g) The comparison of energy dispersion for (e) Pt and $\mathrm{Au}$, and (g) Pt and Pd. (f,h) The comparison of PBC distribution along high symmetry lines for (f) Pt and Au and (h) Pt and Pd. The red, blue, and green curves in $(\mathrm{d}-\mathrm{h})$ represent $\mathrm{Pt}, \mathrm{Pd}$, and $\mathrm{Au}$, respectively.

$$
F_{x y}^{\mathrm{o}}(\vec{k})=2 \operatorname{Im} \sum_{E_{\mathrm{n}}<E_{\text {occ }}} \sum_{m \neq n} \frac{\left\langle n \vec{k}\left|v_{x}\right| m \vec{k}\right\rangle\left\langle m \vec{k}\left|v_{y}\right| n \vec{k}\right\rangle}{\left(E_{n \vec{k}}-E_{m \vec{k}}\right)^{2}}
$$

in which $v_{i}(i=x, y)$ indicates velocity operator and $|n \vec{k}\rangle$ is the eigenvector of the Hamiltonian $H$ with the eigenvalue $E_{n \vec{k}}$, it is easy to find the important characteristic that the size of the Berry curvature can reflect the strength of entanglements of the states at different bands and transversal current. Therefore, we can try to extract another parameter from the Berry curvature that satisfies the requirement to reflect these two properties and even with respect to a time reversal symmetry. With this target, we defined the new parameter as

$$
F_{x y}(\vec{k})=2 \operatorname{Im} \sum_{E_{\mathrm{n}}<E_{\text {occ }}} \sum_{m \neq n} \frac{\left\langle n \vec{k}\left|v_{x}^{\prime}\right| m \vec{k}\right\rangle\left\langle m \vec{k}\left|v_{y}\right| n \vec{k}\right\rangle}{\left(E_{n \vec{k}}-E_{m \vec{k}}\right)^{2}}
$$

with $v_{x}^{\prime}=\left\{p v_{x}\right\}$ and $p=\left(\begin{array}{ll}1 & 0 \\ 0 & 0\end{array}\right)$. Through the project operator $p$, only half of the wave function is used. Equation 2 becomes even with respect to time reversal operation, but it can still reflect the band entanglements and transversal carrier current. Analogous to the ordinary Berry curvature, we call $F_{x y}$ and the module of the integral $\gamma=\left|\int_{\vec{k}} F_{x y}(\vec{k}) d \vec{k}\right|$ as the projected Berry curvature (PBC) and projected Berry phase (PBP), respectively.

As a fundamental understanding, we would like to choose all the well-known transition-metal catalysts as the objects in this work. To see the possible correlation between the PBP and the efficiency of the catalyst, we collected the exchange current densities of HER of all the nonmagnetic catalysts among transition metals. The summarized exchange current densities are given in Figure 2a as a function of metal-hydrogen bond strength, which behaves like the famous volcano-type picture. Following eq 2, the ab-initio calculations of the PBP were performed for all the selected transition metals. The source of the exchange current densities used here and the calculation details can be found in Supporting Information. It can be seen roughly that the catalyst with a large PBP sits on the top position of the volcano plot. Further, a good linear relation between the exchange current density and the PBP is found after considering the symmetry constraint.

As shown in Figure $2 \mathrm{a}-\mathrm{c}$, all the selected transition metals can be classified into three space groups $P 6_{3} / m m c$ (no. 194), $F m \overline{3} m$ (no. 225), and $\operatorname{Im} \overline{3} m$ (no. 229). For the selected elements in each space group, PBP and HER exchange current have a good linear relation, see Figure $2 \mathrm{~b}$. Although the strictly mathematical logic is not clear so far, the linear relation should not be an accidental result. It reflects an important relation between transversal current and surface chemical reaction. From Figure $2 \mathrm{~b}$ and the linear fitting formulas eqs S4-S6 in Supporting Information, one can see that the slopes of the HER exchange current to the PBP are different for each space group, which implies that the crystal structure plays an important role in the understanding of catalysis. The experimental measurements are strongly dependent on the details of the environment, which will introduce unexpected extrinsic effects. The fixed slope for a given space group illustrates that the extrinsic contributions should be similar to the transition metals in the same group. Meanwhile, the difference of each slope implies that the details of extrinsic contributions also vary among different groups.

Besides the metal-hydrogen bond strength, the catalytic efficiency can be also described by the work function and the d-band center. Therefore, PBP and work function (or d-band center) should follow a similar relation. Taking the work function as an example, Figure $2 c$ shows the PBP as a function of collected work functions from experimental studies. As expected, the relationship between PBP and work function is 
still a linear relation for all three groups. Because the work function is strongly dependent on the location of the Fermi energy, we can expect more information about PBP after taking the Fermi level into consideration.

In order to explore the Fermi energy effect, we compare the energy-dependent PBP of the adjacent elements with only one electron difference. Because Pt is believed to be a state-of-theart catalyst, we take $\mathrm{Pt}$ and its neighbor $\mathrm{Au}$, which is considered to be a poor catalyst from both experimental and theoretical points of view, as an example. ${ }^{4}$ From the PBP which is a function of the Fermi energy and shown in Figure 2d, one can easily see that the Fermi energy-dependent PBP for Pt and $\mathrm{Au}$ almost share the same shape, only with a shift of one electron in the energy space.

Because there is only one electron difference for $\mathrm{Au}$ and $\mathrm{Pt}$, the band structure of $\mathrm{Au}$ can almost overlap with Pt after shifting the Fermi level about one electron, as presented in Figure 2e. Because of the difference of the band structure at the Fermi level, the distributions of $\mathrm{PBC}$ are completely different. Owing to the strong band entanglement induced by a small band gap around the Fermi level, the PBC of Pt forms peaks around the high symmetry points of $X$ and $L$. However, the strong band entanglement does not exist anymore after shifting the Fermi energy. This will lead to the almost disappearance of the peak values in $\mathrm{Au}$ (see Figure $2 \mathrm{f}$ ). The huge difference of $\mathrm{PBC}$ distribution in reciprocal space is directly caused by the difference of the PBP after calculating the integral. Therefore, the effect of the chemical potential location is consistent with the d-band center and the work functions.

In addition to the location of chemical potential, we also find some other parameters that can influence the magnitude of the PBP. These parameters can be qualitatively identified by a comparison between two elements in the same column with the same space group. For convenience, we also take $\mathrm{Pt}$ as an example. The other element is its neighbor Pd in the same column. From Figure 2d, we can see that the PBPs of Pt and Pd both have peaks and share the same trend around the charge neutral point. This similarity should be a result of a similar chemical environment of their shell electrons.

To observe their similarities and differences, we also compare their energy dispersion and $\mathrm{PBC}$ along the high symmetry lines. As shown in Figure $2 \mathrm{~g}$, Pt and Pd have similar energy dispersions around the Fermi energy. However, owing to the difference of spin-orbit coupling strength, atomic diameter, and some other details, there are some tiny differences in their energy dispersions. Therefore, Pt cannot overlap with Pd nor Au with a shifted Fermi level. These tiny differences result in the differences of PBC distribution in reciprocal space. Owing to the strong band entanglement which is induced by a small band gap, both Pt and Pd show peaks in PBC around the high symmetry points $L$ and $X$ (see Figure 2h). Furthermore, almost all the peaks of PBC in Pt are positive, whereas $\mathrm{Pd}$ displays a negative peak around the $X$ point and the widths of these peaks are relatively narrow when compared with Pt. The differences of $\mathrm{PBC}$ distribution lead to a small peak in PBP for Pd, which is also in agreement with the difference of the catalytic efficiency for Pt and Pd. ${ }^{4}$ Although the shell electrons of the elements in the same column of the periodic table have a similar chemical environment, the difference of spin-orbit coupling strength and atomic diameter have a strong influence on their intrinsic contributions.
After establishing the linear relation between exchange current density and PBP, one can try to expect the catalytic efficiency via the PBP with the symmetry constraint. Because $\mathrm{Pt}$ is the best catalyst as is known, we use the space group $F m \overline{3} m$ as the example to show the possible candidates of HER catalysts. First of all, the PBP values $\gamma$ of the compound with space group $F m \overline{3} m$, whose structures are downloaded from the Inorganic Crystal Structure Database (ICSD, http://www2.fizkarlsruhe.de/icsd home.html), ${ }^{43}$ are calculated with the same method. Setting the $\gamma$ values of these aim compounds as the input, we can get the exchange current densities (purple dots in Figure 3) using eq S6 in Supporting Information. From the

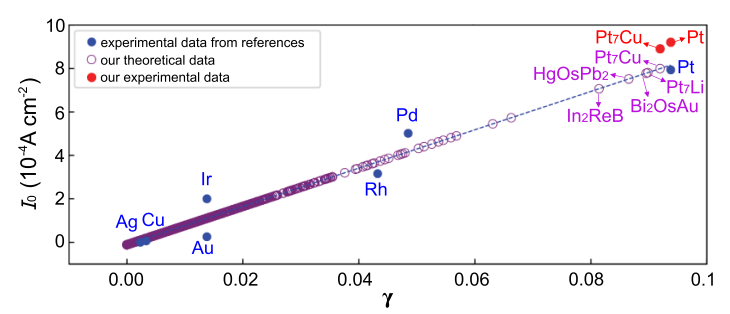

Figure 3. Theoretical result (purple hollow dots) of PBP vs exchange current density for the compounds with space group $F m \overline{3} m$. Solid blue points are obtained from the references listed in Table S2 of Supporting Information. The blue dot line is the fitting model in Figure $2 \mathrm{~b}$. The red solid points are experimental results in our work. $\mathrm{Pt}_{7} \mathrm{Cu}$ is the superlative one and has been verified experimentally.

comparison, one can easily see that the record of $\mathrm{Pt}$ is not broken even after scanning all the compounds, which also illustrates the fact that Pt is the most popular HER catalyst. In the end, we find some good candidates between Pd and Pt. If these compounds are chemically stable, we can expect good catalytic efficiency from them.

To confirm the validity of the prediction based on the proposed descriptor, a candidate of cubic $\mathrm{Pt}_{7} \mathrm{Cu}$ that may carry a large PBP was synthesized and assessed as the HER catalyst. As shown in Figure $4 \mathrm{a}$, the $\mathrm{Pt}_{7} \mathrm{Cu}$ catalyst was deposited on carbon paper directly by a solvothermal method (experimental details can be seen in the Supporting Information). SEM images shown in Figure 4a revealed that the obtained products were mainly well-dispersed nanoparticles. The phase and crystallinity of the as-prepared $\mathrm{Pt}_{7} \mathrm{Cu}$ catalyst were verified by powder X-ray diffraction (XRD) in Figure $4 b$. The diffraction pattern of the product is in good agreement with those of a standard calculated fcc $\mathrm{Pt}_{7} \mathrm{Cu}$ structure (mp-12608 from materialsproject.org and Figure S1 in Supporting Information). The absence of XRD reflections at $43.3^{\circ}$ and $50.4^{\circ}$ for cubic $\mathrm{Cu}$ metal and $39.2^{\circ}$ and $45.6^{\circ}$ for cubic Pt metal signified the successful insertion of $\mathrm{Cu}$ in the $\mathrm{Pt}$ lattice. This is further confirmed by the energy-dispersive spectroscopy (EDS) analysis, which suggests a ratio of $\mathrm{Pt}$ to $\mathrm{Cu}$ closing to 7 (Figure 4c).

HER performance of the $\mathrm{Pt}_{7} \mathrm{Cu}$ catalyst was explored carefully in $0.5 \mathrm{M} \mathrm{H}_{2} \mathrm{SO}_{4}$ solution and compared with the commercial $20 \% \mathrm{Pt} / \mathrm{C}$ catalyst. The equilibrium potential of the HER is obtained by using the Nernst equation before the data analysis, which is determined to be $-0.01 \mathrm{~V}$ in our working conditions (details can be seen in the Supporting Information). ${ }^{44}$ The potential of the $\mathrm{Ag} / \mathrm{AgCl}$ reference electrode is calibrated before usage. The $\mathrm{pH}$ value of 0.5 $\mathrm{H}_{2} \mathrm{SO}_{4}$ is about 0.2 . As exhibited in the HER polarization and Tafel analysis curves (Figure $4 \mathrm{~d}, \mathrm{e}$ ), the $\mathrm{Pt}_{7} \mathrm{Cu}$ catalyst showed 
a
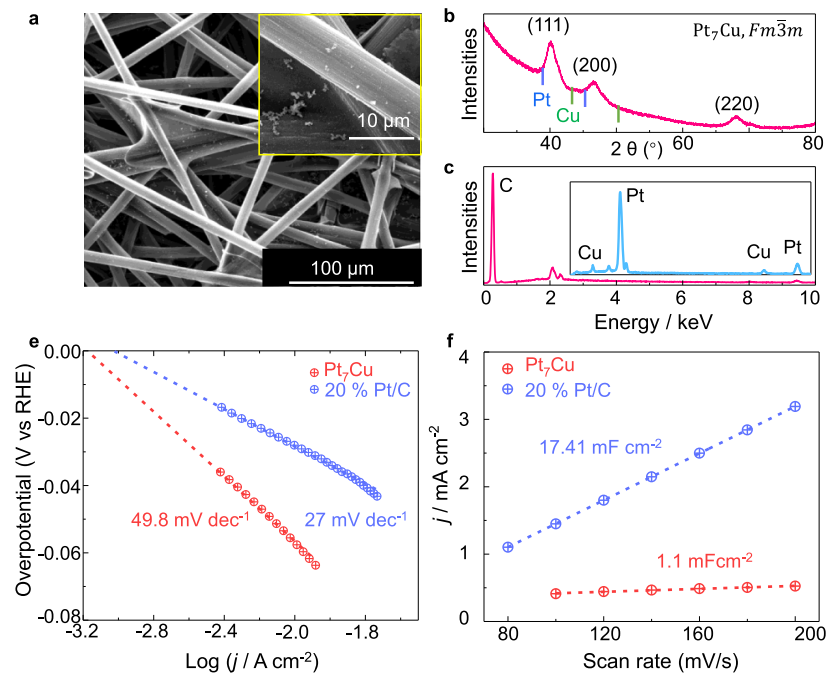
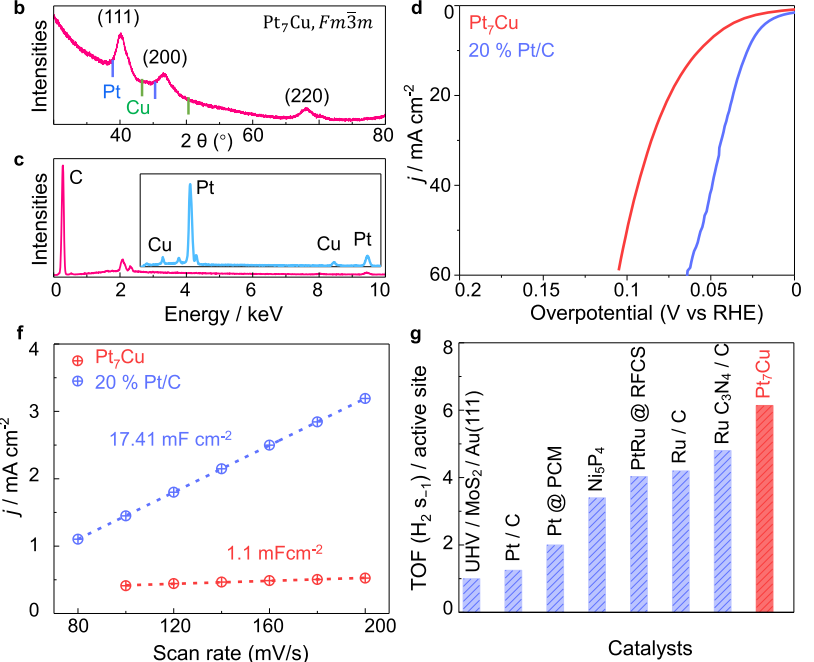

Figure 4. (a) SEM images of the $\mathrm{Pt}_{7} \mathrm{Cu}$ catalyst deposited on carbon paper. (b) X-ray powder diffraction pattern of the as-prepared sample. No peaks belonged to metal $\mathrm{Pt}$ and $\mathrm{Cu}$ are observed. (c) EDS spectrum of the catalyst. The red line was recorded on the carbon paper, while the blue line is obtained from the powder sample. (d,e) HER polarization curves and the corresponding Tafel slopes of the $\mathrm{Pt} \mathrm{Cu}_{7} \mathrm{and} \mathrm{Pt} / \mathrm{C}$ catalysts. (f) Capacitive current densities as a function of scan rate for $\mathrm{Pt}_{7} \mathrm{Cu}$ and $\mathrm{Pt} / \mathrm{C}$ catalysts. $(\mathrm{g}) \mathrm{Comparison}$ of $\mathrm{TOF}$ values of the $\mathrm{Pt} \mathrm{Cu}_{7} \mathrm{Cu}$ a $\mathrm{Pt} / \mathrm{C}$ with other recently reported benchmarks HER electrocatalysts.

a very small overpotential of $56 \mathrm{mV}$ at a current density of 10 $\mathrm{mA} \mathrm{cm}{ }^{-2}$ along with a Tafel slope of only $49.8 \mathrm{mV} \mathrm{dec}^{-1}$. The overpotential for the $\mathrm{Pt} / \mathrm{C}$ catalyst to deliver the same current density is $29 \mathrm{mV}$ with the value of Tafel slope as low as $27 \mathrm{mV}$ $\operatorname{dec}^{-1}$. The difference in the measured activity may be caused by the low number of exposed active sites as evidenced by the low double-layer capacitance of the $\mathrm{Pt}_{7} \mathrm{Cu}$ catalyst (Figure $4 \mathrm{f}$ ). The linear part of the Tafel slopes is extrapolated to an overpotential of $0 \mathrm{~V}$ to obtain the corresponding exchange current densities. ${ }^{45}$ The exchange current density of the $\mathrm{Pt}_{7} \mathrm{Cu}$ catalyst was determined to be by $0.74 \mathrm{~mA} \mathrm{~cm}^{-2}$ by extrapolating the Tafel plots (Figure 4e). This value is close to that of the $\mathrm{Pt} / \mathrm{C}$ catalyst at acidic conditions $(0.92 \mathrm{~mA}$ $\mathrm{cm}^{-2}$ ), indicating high electrocatalytic activity of the $\mathrm{Pt}_{7} \mathrm{Cu}$ catalyst.

The turnover frequency (TOF), which is believed as the best figure-of-merit for a fair evaluation of different catalysts, was obtained from the polarization curve (details can be seen in the Supporting Information). The double-layer capacitance $\left(C_{\mathrm{dl}}\right)$ were estimated from the cyclic voltammetry curves at different scan rates first. As displayed in Figure $4 \mathrm{f}$, the values $C_{\mathrm{dl}}$ are calculated to be 1.1 and $17.4 \mathrm{mF} \mathrm{cm}^{-2}$ for $\mathrm{Pt}_{7} \mathrm{Cu}$ and $\mathrm{Pt} / \mathrm{C}$ catalysts, respectively. This suggests that the $\mathrm{Pt} / \mathrm{C}$ commercial catalysts display more catalytically active sites in comparison to the $\mathrm{Pt}_{7} \mathrm{Cu}$ sample. The $\mathrm{Pt}_{7} \mathrm{Cu}$ catalyst achieves a TOF value of $6.14 \mathrm{~S}^{-1}$ at an overpotential of $100 \mathrm{mV}$. This value is larger than that of the benchmark noble metal or transition-metal based catalysts as displayed in Figure $4 \mathrm{~g}$, indicating that the $\mathrm{Pt}_{7} \mathrm{Cu}$ catalyst has an high intrinsic catalytic activity, which is consistent with the prediction based on the PBP theory. ${ }^{46-49}$

\section{CONCLUSIONS}

In summary, with the proposed parameter PBP, we systematically studied the bulk intrinsic effect for the electronic structure of transition-metal HER catalysts. It was found that the PBP and the exchange current for HER has a linear relation after taking the symmetry constraint into consideration. For a given crystal structure, the effect of extrinsic contribution was very similar to the catalysts with the same group and varied significantly with the catalysts with different groups. The analysis of the PBC of neighboring elements with the same space group illustrated the importance of the Fermi level location, which agrees with the previous d-band center and work function models. Meanwhile, the spin-orbit coupling and atom diameters are also important parameters that influence the intrinsic bulk contributions.

\section{ASSOCIATED CONTENT}

SI Supporting Information

The Supporting Information is available free of charge at https://pubs.acs.org/doi/10.1021/acscatal.9b05539.

Details about materials synthesis, electrocatalytic characterization; electrochemical active surface area and TOF calculation estimation; and analysis of exchange current density and work function data linear fitting (PDF)

\section{AUTHOR INFORMATION}

\section{Corresponding Authors}

Yan Sun - Max Planck Institute for Chemical Physics of Solids, 01187 Dresden, Germany; Email: Yan.Sun@cpfs.mpg.de

Claudia Felser - Max Planck Institute for Chemical Physics of Solids, 01187 Dresden, Germany; Center for Nanoscale Systems, Faculty of Arts and Sciences, Harvard University, Cambridge, Massachusetts 02138, United States;

Email: Claudia.Felser@cpfs.mpg.de

\section{Authors}

Qiunan Xu - Max Planck Institute for Chemical Physics of

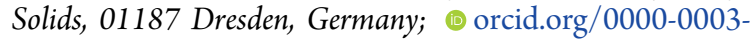
3974-5037

Guowei Li - Max Planck Institute for Chemical Physics of Solids, 01187 Dresden, Germany; 이이이.org/0000-00031837-3967

Yang Zhang - Max Planck Institute for Chemical Physics of Solids, 01187 Dresden, Germany

Qun Yang - Max Planck Institute for Chemical Physics of Solids, 01187 Dresden, Germany 
Complete contact information is available at:

https://pubs.acs.org/10.1021/acscatal.9b05539

\section{Author Contributions}

Q.X. and G.L. contribute equally to this work. C.F., Y.S., and G.L. conceived the study. Q.X., Q.Y., and Y.Z. performed the calculations. Q.X. and Y.S. analyzed the data and provided the plots. G.L. and Y.S. wrote the manuscript with all co-authors.

\section{Notes}

The authors declare no competing financial interest.

\section{ACKNOWLEDGMENTS}

This work was financially supported by the ERC Advanced grant no. 291472 "Idea Heusler" and ERC Advanced grant no. 742068 "TOPMAT". We acknowledge funding by the DFG through SFB 1143 (project ID 247310070) and the Wuerzburg-Dresden Cluster of Excellence on Complexity and Topology in Quantum Matter-ct.qmat (EXC 2147, project ID 39085490).

\section{REFERENCES}

(1) Liu, Y.; Wu, J.; Hackenberg, K. P.; Zhang, J.; Wang, Y. M.; Yang, Y.; Keyshar, K.; Gu, J.; Ogitsu, T.; Vajtai, R.; Lou, J.; Ajayan, P. M.; Wood, B. C.; Yakobson, B. I. Self-optimizing, highly surface-active layered metal dichalcogenide catalysts for hydrogen evolution. Nat. Energy 2017, 2, 17127.

(2) Kuhn, A. T.; Mortimer, C. J.; Bond, G. C.; Lindley, J. A critical analysis of correlations between the rate of the electrochemical hydrogen evolution reaction and physical properties of the elements. J. Electroanal. Chem. Interfacial Electrochem. 1972, 34, 1-14.

(3) Swarup, J. Dependence of hydrogen overvoltage on the melting points of cathode metal. Indian J. Chem. 1969, 7, 896-900.

(4) Trasatti, S. Work function, electronegativity, and electrochemical behaviour of metals. J. Electroanal. Chem. Interfacial Electrochem. 1972, 39, 163-184.

(5) Butler, J. A. V. The Theory of the Hydrogen Overvoltage. J. Chem. Phys. 1941, 9, 279-280.

(6) Parsons, R. The rate of electrolytic hydrogen evolution and the heat of adsorption of hydrogen. Trans. Faraday Soc. 1958, 54, 10531063.

(7) Greeley, J.; Jaramillo, T. F.; Bonde, J.; Chorkendorff, I.; Nørskov, J. K. Computational high-throughput screening of electrocatalytic materials for hydrogen evolution. Nat. Mater. 2006, 5, 909-913.

(8) Lundqvist, B. I.; Gunnarsson, O.; Hjelmberg, H.; Nørskov, J. K. Theoretical description of molecule-metal interaction and surface reactions. Surf. Sci. 1979, 89, 196-225.

(9) Norskov, J. Theory of chemisorption and heterogeneous catalysis. Phys. B 1984, 127, 193-202.

(10) Vayenas, C. G.; Bebelis, S.; Ladas, S. Dependence of catalytic rates on catalyst work function. Nature 1990, 343, 625-627.

(11) Hammer, B.; Morikawa, Y.; Nørskov, J. K. CO chemisorption at metal surfaces and overlayers. Phys. Rev. Lett. 1996, 76, 2141.

(12) Nørskov, J. K.; Rossmeisl, J.; Logadottir, A.; Lindqvist, L.; Kitchin, J. R.; Bligaard, T.; Jonsson, H. Origin of the overpotential for oxygen reduction at a fuel-cell cathode. J. Phys. Chem. B 2004, 108, 17886-17892.

(13) Norsko, J. K. Chemisorption on metal surfaces. Rep. Prog. Phys. 1990, 53, 1253.

(14) Hammer, B.; Norskov, J. K. Why gold is the noblest of all the metals. Nature 1995, 376, 238

(15) Hammer, B.; Nørskov, J. K. Electronic factors determining the reactivity of metal surfaces. Surf. Sci. 1995, 343, 211.

(16) Xin, H.; Vojvodic, A.; Voss, J.; Nørskov, J. K.; Abild-Pedersen, F. Effects of d-band shape on the surface reactivity of transition-metal alloys. Phys. Rev. B: Condens. Matter Mater. Phys. 2014, 89, 115114.
(17) Quaino, P.; Juarez, F.; Santos, E.; Schmickler, W. Volcano plots in hydrogen electrocatalysis-uses and abuses. Beilstein J. Nanotechnol. 2014, 5, 846.

(18) Zeradjanin, A. R.; Grote, J.-P.; Polymeros, G.; Mayrhofer, K. J. J. A Critical Review on Hydrogen Evolution Electrocatalysis: Reexploring the Volcano-relationship. Electroanalysis 2016, 28, 22562269.

(19) Seh, Z. W.; Kibsgaard, J.; Dickens, C. F.; Chorkendorff, I.; Nørskov, J. K.; Jaramillo, T. F. Combining theory and experiment in electrocatalysis: Insights into materials design. Science 2017, 355, No. eaad4998.

(20) Zegkinoglou, I.; Zendegani, A.; Sinev, I.; Kunze, S.; Mistry, H.; Jeon, H. S.; Zhao, J.; Hu, M. Y.; Alp, E. E.; Piontek, S.; Smialkowski, M.; Apfel, U.-P.; Körmann, F.; Neugebauer, J.; Hickel, T.; Roldan Cuenya, B. Operando Phonon Studies of the Protonation Mechanism in Highly Active Hydrogen Evolution Reaction Pentlandite Catalysts. J. Am. Chem. Soc. 2017, 139, 14360-14363.

(21) Koper, M. T. M. Analysis of electrocatalytic reaction schemes: distinction between rate-determining and potential-determining steps. J. Solid State Chem. 2013, 17, 339-344.

(22) Exner, K. S.; Over, H. Kinetics of Electrocatalytic Reactions from First-Principles: $\mathrm{A}$ Critical Comparison with the $\mathrm{Ab}$ Initio Thermodynamics Approach. Acc. Chem. Res. 2017, 50, 1240-1247.

(23) Hellstern, T. R.; Kibsgaard, J.; Tsai, C.; Palm, D. W.; King, L. A.; Abild-Pedersen, F.; Jaramillo, T. F. Investigating Catalyst-Support Interactions To Improve the Hydrogen Evolution Reaction Activity of Thiomolybdate $\left[\mathrm{Mo}_{3} \mathrm{~S}_{13}\right]^{2-}$ Nanoclusters. ACS Catal. 2017, 7, 71267130.

(24) Ooka, H.; Nakamura, R. Shift of the Optimum Binding Energy at Higher Rates of Catalysis. J. Phys. Chem. Lett. 2019, 10, 67066713.

(25) Exner, K. S. Is Thermodynamics a Good Descriptor for the Activity? Re-Investigation of Sabatier's Principle by the Free Energy Diagram in Electrocatalysis. ACS Catal. 2019, 9, 5320-5329.

(26) Exner, K. S.; Heß, F.; Over, H.; Seitsonen, A. P. Combined experiment and theory approach in surface chemistry: Stairway to heaven? Surf. Sci. 2015, 640, 165-180.

(27) Su, J.; Yang, Y.; Xia, G.; Chen, J.; Jiang, P.; Chen, Q. Ruthenium-cobalt nanoalloys encapsulated in nitrogen-doped graphene as active electrocatalysts for producing hydrogen in alkaline media. Nat. Commun. 2017, 8, 14969.

(28) Voiry, D.; Yamaguchi, H.; Li, J.; Silva, R.; Alves, D. C. B.; Fujita, T.; Chen, M.; Asefa, T.; Shenoy, V. B.; Eda, G.; Chhowalla, M. Enhanced catalytic activity in strained chemically exfoliated WS 2 nanosheets for hydrogen evolution. Nat. Mater. 2013, 12, 850.

(29) Li, Y.; Wang, H.; Xie, L.; Liang, Y.; Hong, G.; Dai, H. MoS nanoparticles grown on graphene: an advanced catalyst for the hydrogen evolution reaction. J. Am. Chem. Soc. 2011, 133, 72967299.

(30) Chen, H.; Zhu, W.; Xiao, D.; Zhang, Z. CO oxidation facilitated by robust surface states on Au-covered topological insulators. Phys. Rev. Lett. 2011, 107, 056804.

(31) Rajamathi, C. R.; Gupta, U.; Kumar, N.; Yang, H.; Sun, Y.; Süß, V.; Shekhar, C.; Schmidt, M.; Blumtritt, H.; Werner, P.; Yan, B.; Parkin, S.; Felser, C.; Rao, C. N. R. Weyl semimetals as hydrogen evolution catalysts. Adv. Mater. 2017, 29, 1606202.

(32) Politano, A.; Chiarello, G.; Li, Z.; Fabio, V.; Wang, L.; Guo, L.; Chen, X.; Boukhvalov, D. W. Toward the Effective Exploitation of Topological Phases of Matter in Catalysis: Chemical Reactions at the Surfaces of $\mathrm{NbAs}$ and TaAs Weyl Semimetals. Adv. Funct. Mater. 2018, 28, 1800511.

(33) Rajamathi, C. R.; Gupta, U.; Pal, K.; Kumar, N.; Yang, H.; Sun, Y.; Shekhar, C.; Yan, B.; Parkin, S.; Waghmare, U. V.; Felser, C.; Rao, C. N. R. Photochemical water splitting by bismuth chalcogenide topological insulators. Chemphyschem 2017, 18, 2322-2327.

(34) Li, J.; Ma, H.; Xie, Q.; Feng, S.; Ullah, S.; Li, R.; Dong, J.; Li, D.; Li, Y.; Chen, X.-Q. Topological quantum catalyst: Dirac nodal line states and a potential electrocatalyst of hydrogen evolution in the TiSi family. Sci. China Mater. 2018, 61, 23-29. 
(35) Yan, B.; Stadtmüller, B.; Haag, N.; Jakobs, S.; Seidel, J.; Jungkenn, D.; Mathias, S.; Cinchetti, M.; Aeschlimann, M.; Felser, C. Topological states on the gold surface. Nat. Commun. 2015, 6, 10167.

(36) Schoop, L. M.; Pielnhofer, F.; Lotsch, B. V. Chemical Principles of Topological Semimetals. Chem. Mater. 2018, 30, 3155-3176.

(37) Bentley, C. L.; Kang, M.; Maddar, F. M.; Li, F.; Walker, M.; Zhang, J.; Unwin, P. R. Electrochemical maps and movies of the hydrogen evolution reaction on natural crystals of molybdenite $\left(\mathrm{MoS}_{2}\right)$ : basal vs. edge plane activity. Chem. Sci. 2017, 8, 6583-6593.

(38) Xiao, D.; Chang, M.-C.; Niu, Q. Berry phase effects on electronic properties. Rev. Mod. Phys. 2010, 82, 1959-2007.

(39) Matthews, D. B. Ph.D. Thesis, University of Pennsylvania, 1965.

(40) Parsons, R. Handbook of Electrochemical Constants; Elsevier, 1959.

(41) Cheng, Y.; Fan, X.; Liao, F.; Lu, S.; Li, Y.; Liu, L.; Li, Y.; Lin, H.; Shao, M.; Lee, S.-T. Os/Si nanocomposites as excellent hydrogen evolution electrocatalysts with thermodynamically more favorable hydrogen adsorption free energy than platinum. Nano Energy 2017, 39, 284-290.

(42) Drummond, T. J. Work Functions of the Transition Metals and Metal Silicides; University of North Texas Libraries, UNT Digital Library: Albuquerque, New Mexico, 1999.

(43) Hellenbrandt, M. The Inorganic Crystal Structure Database (ICSD)-Present and Future. Crystallogr. Rev. 2004, 10, 17.

(44) Sohrabnejad-Eskan, I.; Goryachev, A.; Exner, K. S.; Kibler, L. A.; Hensen, E. J. M.; Hofmann, J. P.; Over, H. TemperatureDependent Kinetic Studies of the Chlorine Evolution Reaction over $\mathrm{RuO}_{2}(110)$ Model Electrodes. ACS Catal. 2017, 7, 2403-2411.

(45) Exner, K. S.; Sohrabnejad-Eskan, I.; Over, H. A universal approach to determine the free energy diagram of an electrocatalytic reaction. ACS Catal. 2018, 8, 1864-1879.

(46) Zhang, H.; An, P.; Zhou, W.; Guan, B. Y.; Zhang, P.; Dong, J.; Lou, X. W. Dynamic traction of lattice-confined platinum atoms into mesoporous carbon matrix for hydrogen evolution reaction. Sci. Adv. 2018, 4, No. eaao6657.

(47) Li, K.; Li, Y.; Wang, Y.; Ge, J.; Liu, C.; Xing, W. Enhanced electrocatalytic performance for the hydrogen evolution reaction through surface enrichment of platinum nanoclusters alloying with ruthenium in situ embedded in carbon. Energy Environ. Sci. 2018, 11, $1232-1239$.

(48) Kibsgaard, J.; Jaramillo, T. F.; Besenbacher, F. Building an appropriate active-site motif into a hydrogen-evolution catalyst with thiomolybdate $\left[\mathrm{Mo}_{3} \mathrm{~S}_{13}\right]^{2-}$ clusters. Nat. Chem. 2014, 6, 248-253.

(49) Laursen, A. B.; Patraju, K. R.; Whitaker, M. J.; Retuerto, M.; Sarkar, T.; Yao, N.; Ramanujachary, K. V.; Greenblatt, M.; Dismukes, G. C. Nanocrystalline Ni5P4: a hydrogen evolution electrocatalyst of exceptional efficiency in both alkaline and acidic media. Energy Environ. Sci. 2015, 8, 1027-1034. 